\title{
Some Formulas for New Quadruple Hypergeometric Functions
}

\author{
Jihad A. Younis $\mathbb{D}^{1}{ }^{1}$ Hassen Aydi $\mathbb{D}^{2,3,4}$ and Ashish Verma ${ }^{5}$ \\ ${ }^{1}$ Department of Mathematics, Aden University, Aden, Yemen \\ ${ }^{2}$ Universite de Sousse, Institut Superieur d'Informatique et des Techniques de Communication, H. Sousse 4000, Tunisia \\ ${ }^{3}$ Department of Mathematics and Applied Mathematics, Sefako Makgatho Health Sciences University, \\ Ga-Rankuwa, South Africa \\ ${ }^{4}$ China Medical University Hospital, China Medical University, Taichung 40402, Taiwan \\ ${ }^{5}$ Department of Mathematics, V.B.S. Purvanchal University, Jaunpur, India
}

Correspondence should be addressed to Hassen Aydi; hassen.aydi@isima.rnu.tn

Received 9 February 2021; Revised 10 March 2021; Accepted 16 March 2021; Published 1 April 2021

Academic Editor: Ahmet Ocak Akdemir

Copyright (c) 2021 Jihad A. Younis et al. This is an open access article distributed under the Creative Commons Attribution License, which permits unrestricted use, distribution, and reproduction in any medium, provided the original work is properly cited.

In this paper, we aim to introduce six new quadruple hypergeometric functions. Then, we investigate certain formulas and representations for these functions such as symbolic formulas, differential formulas, and integral representations.

\section{Introduction}

Hypergeometric functions of several variables play an important role in diverse areas of science and engineering. The developments in applied mathematics, mathematical physics, chemistry, combinatorics, statistics, numerical analysis, and other areas have led to increasing interest in the study of multiple hypergeometric functions. Many authors have studied a number of formulas involving hypergeometric functions (see, e.g., [1-6]).

In [7], Exton presented twenty-one complete hypergeometric functions in four variables denoted by symbols $K_{1}, K_{2}, \ldots, K_{21}$. In [8], Sharma and Parihar defined eightythree complete quadruple hypergeometric functions, namely, $F_{1}^{(4)}, F_{2}^{(4)}, \ldots, F_{83}^{(4)}$. Bin-Saad and Younis [9] gave thirty new quadruple hypergeometric functions given by $X_{1}^{(4)}, X_{2}^{(4)}, \ldots, X_{30}^{(4)}$. In [10], the authors discovered the existence of twenty additional complete hypergeometric functions in four variables $X_{31}^{(4)}, X_{32}^{(4)}, \ldots, X_{50}^{(4)}$. Each quadruple hypergeometric function in [7-10] is of the form

$$
X^{(4)}(.)=\sum_{m, n, p, q=0}^{\infty} \Omega(m, n, p, q) \frac{x^{m}}{m !} \frac{y^{n}}{n !} \frac{z^{p}}{p !} \frac{u^{q}}{q !},
$$

where $\Omega(m, n, p, q)$ is a certain sequence of complex parameters, and there are twelve parameters in each series of $X^{(4)}\left(\right.$.) (eight $a^{\prime} s$ and four $c^{\prime} s$ ). The 1st, 2 nd, 3rd, and 4 th parameters in $X^{(4)}($.$) are connected with integers$ $m, n, p$, and $q$, respectively. Each repeated parameter in the series $X^{(4)}($.) points out a term with double parameters in $\Omega(m, n, p, q)$. For example, $X^{(4)}\left(a_{1}, a_{1}, a_{2}, a_{2}\right.$, $\left.a_{3}, a_{3}, a_{4}, a_{5}\right)$ means that $\left(a_{1}\right)_{m+n}\left(a_{2}\right)_{p+q}\left(a_{3}\right)_{m+n}\left(a_{4}\right)_{p}\left(a_{5}\right)_{q}$ includes the term. Similarly, $X^{(4)}\left(a_{1}, a_{1}, a_{1}, a_{2}, a_{1}\right.$, $\left.a_{1}, a_{2}, a_{3}\right)$ points out the term $\left(a_{1}\right)_{2 m+2 n+p}\left(a_{2}\right)_{p+q}\left(a_{3}\right)_{q}$, and $X^{(4)}\left(a_{1}, a_{1}, a_{2}, a_{3}, a_{1}, a_{2}, a_{3}, a_{4}\right)$ shows the existence of the term $\left(a_{1}\right)_{2 m+n}\left(a_{2}\right)_{n+p}\left(a_{3}\right)_{p+q}\left(a_{4}\right)_{q}$. Thus, it is possible to form various combinations of indices. There seems to be no way of independently establishing the number of distinct Gaussian hypergeometric series for any given integer $n \geq 2$ without explicitly stating all such series. Thus, in every situation with $n=4$, one ought to begin by actually constructing the set just as in the case $n=3$ (see [11]).

By using the conventions and notations above, we now introduce further quadruple hypergeometric functions as follows: 


$$
\begin{aligned}
& X_{85}^{(4)}\left(a_{1}, a_{1}, a_{2}, a_{3}, a_{1}, a_{2}, a_{3}, a_{4} ; c_{1}, c_{2}, c_{3}, c_{1} ; x, y, z, u\right)=\sum_{m, n, p, q=0}^{\infty} \frac{\left(a_{1}\right)_{2 m+n}\left(a_{2}\right)_{n+p}\left(a_{3}\right)_{p+q}\left(a_{4}\right)_{q}}{\left(c_{1}\right)_{m+q}\left(c_{2}\right)_{n}\left(c_{3}\right)_{p}} \frac{x^{m}}{m !} \frac{y^{n}}{n !} \frac{z^{p}}{p !} \frac{u^{q}}{q !} \\
& X_{86}^{(4)}\left(a_{1}, a_{1}, a_{2}, a_{3}, a_{1}, a_{2}, a_{3}, a_{4} ; c_{2}, c_{1}, c_{3}, c_{1} ; x, y, z, u\right)=\sum_{m, n, p, q=0}^{\infty} \frac{\left(a_{1}\right)_{2 m+n}\left(a_{2}\right)_{n+p}\left(a_{3}\right)_{p+q}\left(a_{4}\right)_{q}}{\left(c_{1}\right)_{n+q}\left(c_{2}\right)_{m}\left(c_{3}\right)_{p}} \frac{x^{m}}{m !} \frac{y^{n}}{n !} \frac{z^{p}}{p !} \frac{u^{q}}{q !}, \\
& X_{87}^{(4)}\left(a_{1}, a_{1}, a_{2}, a_{3}, a_{1}, a_{2}, a_{3}, a_{4} ; c_{1}, c_{2}, c_{1}, c_{2} ; x, y, z, u\right)==\sum_{m, n, p, q=0}^{\infty} \frac{\left(a_{1}\right)_{2 m+n}\left(a_{2}\right)_{n+p}\left(a_{3}\right)_{p+q}\left(a_{4}\right)_{q}}{\left(c_{1}\right)_{m+p}\left(c_{2}\right)_{n+q}} \frac{x^{m}}{m !} \frac{y^{n}}{n !} \frac{z^{p}}{p !} \frac{u^{q}}{q !} \\
& X_{88}^{(4)}\left(a_{1}, a_{1}, a_{2}, a_{3}, a_{1}, a_{2}, a_{3}, a_{4} ; c_{1}, c_{2}, c_{2}, c_{1} ; x, y, z, u\right)=\sum_{m, n, p, q=0}^{\infty} \frac{\left(a_{1}\right)_{2 m+n}\left(a_{2}\right)_{n+p}\left(a_{3}\right)_{p+q}\left(a_{4}\right)_{q}}{\left(c_{1}\right)_{m+q}\left(c_{2}\right)_{n+p}} \frac{x^{m}}{m !} \frac{y^{n}}{n !} \frac{z^{p}}{p !} \frac{u^{q}}{q !} \\
& X_{89}^{(4)}\left(a_{1}, a_{1}, a_{2}, a_{3}, a_{1}, a_{2}, a_{3}, a_{4} ; c_{1}, c_{2}, c_{1}, c_{1} ; x, y, z, u\right)=\sum_{m, n, p, q=0}^{\infty} \frac{\left(a_{1}\right)_{2 m+n}\left(a_{2}\right)_{n+p}\left(a_{3}\right)_{p+q}\left(a_{4}\right)_{q}}{\left(c_{1}\right)_{m+p+q}\left(c_{2}\right)_{n}} \frac{x^{m}}{m !} \frac{y^{n}}{n !} \frac{z^{p}}{p !} \frac{u^{q}}{q !} \\
& X_{90}^{(4)}\left(a_{1}, a_{1}, a_{2}, a_{3}, a_{1}, a_{2}, a_{3}, a_{4} ; c, c, c, c ; x, y, z, u\right)=\sum_{m, n, p, q=0}^{\infty} \frac{\left(a_{1}\right)_{2 m+n}\left(a_{2}\right)_{n+p}\left(a_{3}\right)_{p+q}\left(a_{4}\right)_{q}}{(c)_{m+n+p+q}} \frac{x^{m}}{m !} \frac{y^{n}}{n !} \frac{z^{p}}{p !} \frac{u^{q}}{q !}
\end{aligned}
$$

for

$$
\left(|x|<\frac{1}{4},|y|<1,|z|<1,|u|<1\right) .
$$

Here, $(a)_{m}$ is the Pochhammer symbol defined (for $a, m \in \mathbb{C}$ ), in terms of the familiar Gamma function $\Gamma$, by (see, e.g., [11], p. 2 and p. 5)

$$
\begin{aligned}
(a)_{m} & :=\frac{\Gamma(a+m)}{\Gamma(a)}, \quad\left(a+m \in \mathbb{C} \backslash \mathbb{Z}_{0}^{-}\right) \\
& = \begin{cases}1, & (m=0), \\
a(a+1) \ldots(a+m-1), & (m=n \in \mathbb{N}),\end{cases}
\end{aligned}
$$

where $\mathbb{C}, \mathbb{Z}_{0}^{-}$, and $\mathbb{N}$ denote the sets of complex numbers, nonpositive integers, and positive integers, respectively.
We recall the Gauss hypergeometric function [12] which is defined by

$$
{ }_{2} F_{1}(a, b ; c ; x)=\sum_{n=0}^{\infty} \frac{(a)_{n}(b)_{n}}{(c)_{n}} \frac{x^{n}}{n !}, \quad(|x|<1) .
$$

Appell's double hypergeometric function $F_{2}$ is defined as follows [13]:

$$
F_{2}(a, b, c ; d, e ; x, y)=\sum_{m, n=0}^{\infty} \frac{(a)_{m+n}(b)_{m}(c)_{n}}{(d)_{m}(e)_{n}} \frac{x^{m}}{m !} \frac{y^{n}}{n !} .
$$

In [14], Exton established twenty distinct triple hypergeometric functions, which are denoted by $X_{1}, X_{2}, \ldots, X_{20}$. We introduce the definitions of five of these functions in the following:

$$
\begin{aligned}
X_{15}\left(a_{1}, a_{2}, a_{3} ; c_{2}, c_{1} ; x, y, z\right) & =\sum_{m, n, p=0}^{\infty} \frac{\left(a_{1}\right)_{2 m+n}\left(a_{2}\right)_{n+p}\left(a_{3}\right)_{p}}{\left(c_{1}\right)_{n+p}\left(c_{2}\right)_{m}} \frac{x^{m}}{m !} \frac{y^{n}}{n !} \frac{z^{p}}{p !} \\
X_{16}\left(a_{1}, a_{2}, a_{3} ; c_{1}, c_{2} ; x, y, z\right) & =\sum_{m, n, p=0}^{\infty} \frac{\left(a_{1}\right)_{2 m+n}\left(a_{2}\right)_{n+p}\left(a_{3}\right)_{p}}{\left(c_{1}\right)_{m+p}\left(c_{2}\right)_{n}} \frac{x^{m}}{m !} \frac{y^{n}}{n !} \frac{z^{p}}{p !}, \\
X_{17}\left(a_{1}, a_{2}, a_{3} ; c_{1}, c_{2}, c_{3} ; x, y, z\right) & =\sum_{m, n, p=0}^{\infty} \frac{\left(a_{1}\right)_{2 m+n}\left(a_{2}\right)_{n+p}\left(a_{3}\right)_{p}}{\left(c_{1}\right)_{m}\left(c_{2}\right)_{n}\left(c_{3}\right)_{p}} \frac{x^{m}}{m !} \frac{y^{n}}{n !} \frac{z^{p}}{p !}, \\
X_{18}\left(a_{1}, a_{2}, a_{3}, a_{4} ; c ; x, y, z\right) & =\sum_{m, n, p=0}^{\infty} \frac{\left(a_{1}\right)_{2 m+n}\left(a_{2}\right)_{n}\left(a_{3}\right)_{p}\left(a_{4}\right)_{p}}{(c)_{m+n+p}} \frac{x^{m}}{m !} \frac{y^{n}}{n !} \frac{z^{p}}{p !}, \\
X_{20}\left(a_{1}, a_{2}, a_{3}, a_{4} ; c_{1}, c_{2} ; x, y, z\right) & =\sum_{m, n, p=0}^{\infty} \frac{\left(a_{1}\right)_{2 m+n}\left(a_{2}\right)_{n}\left(a_{3}\right)_{p}\left(a_{4}\right)_{p}}{\left(c_{1}\right)_{m+p}\left(c_{2}\right)_{n}} \frac{x^{m}}{m !} \frac{y^{n}}{n !} \frac{z^{p}}{p !} .
\end{aligned}
$$


The Lauricella functions of three variables $F_{M}, F_{N}, F_{p}, F_{S}$, and $F_{T}$ are defined in $[11,15]$ :

$$
\begin{aligned}
F_{M}\left(a_{1}, a_{2}, a_{2}, b_{1}, b_{2}, b_{1} ; c_{1}, c_{2}, c_{2} ; x, y, z\right) & =\sum_{m, n, p=0}^{\infty} \frac{\left(a_{1}\right)_{m}\left(a_{2}\right)_{n+p}\left(b_{1}\right)_{m+p}\left(b_{2}\right)_{n}}{\left(c_{1}\right)_{m}\left(c_{2}\right)_{n+p}} \frac{x^{m}}{m !} \frac{y^{n}}{n !} \frac{z^{p}}{p}, \\
F_{N}\left(a_{1}, a_{2}, a_{3}, b_{1}, b_{2}, b_{1} ; c_{1}, c_{2}, c_{2} ; x, y, z\right) & =\sum_{m, n, p=0}^{\infty} \frac{\left(a_{1}\right)_{m}\left(a_{2}\right)_{n}\left(a_{3}\right)_{p}\left(b_{1}\right)_{m+p}\left(b_{2}\right)_{n}}{\left(c_{1}\right)_{m}\left(c_{2}\right)_{n+p}} \frac{x^{m}}{m !} \frac{y^{n}}{n !} \frac{z^{p}}{p}, \\
F_{P}\left(a_{1}, a_{2}, a_{1}, b_{1}, b_{1}, b_{2} ; c_{1}, c_{2}, c_{2} ; x, y, z\right) & =\sum_{m, n, p=0}^{\infty} \frac{\left(a_{1}\right)_{m+p}\left(a_{2}\right)_{n}\left(b_{1}\right)_{m+n}\left(b_{2}\right)_{p}}{\left(c_{1}\right)_{m}\left(c_{2}\right)_{n+p}} \frac{x^{m}}{m !} \frac{y^{n}}{n !} \frac{z^{p}}{p}, \\
F_{S}\left(a_{1}, a_{2}, a_{2}, b_{1}, b_{2}, b_{3} ; c, c, c ; x, y, z\right) & =\sum_{m, n, p=0}^{\infty} \frac{\left(a_{1}\right)_{m}\left(a_{2}\right)_{n+p}\left(b_{1}\right)_{m}\left(b_{2}\right)_{n}\left(b_{3}\right)_{p}}{(c)_{m+n+p}} \frac{x^{m}}{m !} \frac{y^{n}}{n !} \frac{z^{p}}{p}, \\
F_{T}\left(a_{1}, a_{2}, a_{2}, b_{1}, b_{2}, b_{1} ; c, c, c ; x, y, z\right) & =\sum_{m, n, p=0}^{\infty} \frac{\left(a_{1}\right)_{m}\left(a_{2}\right)_{n+p}\left(b_{1}\right)_{m+p}\left(b_{2}\right)_{n}}{(c)_{m+n+p}} \frac{x^{m}}{m !} \frac{y^{n}}{n !} \frac{z^{p}}{p} .
\end{aligned}
$$

The structure of this paper is as follows. In Sections 2 to 5 , we obtain several symbolic formulas, differentiation formulas, operator formulas, and integral representations for the hypergeometric functions of four variables $X_{85}^{(4)}, X_{86}^{(4)}, \ldots, X_{90}^{(4)}$.

\section{Symbolic Formulas}

First of all, we recall the following symbolic operators (see [16]):

$$
\begin{aligned}
& \quad\left[1-\left(D_{\alpha_{1}} D_{\alpha_{2}} \beta^{-1} D_{\beta}^{-1} \gamma^{-1} D_{\gamma}^{-1} \alpha_{1} \alpha_{2}\right) u\right]^{-a} X_{17}\left(a_{1}, a_{2}, a_{3} ; c_{1}, c_{2} ; \beta x, y, \alpha_{1} z\right) \\
& \quad \times\left(\alpha_{1}^{a_{3}-1} \alpha_{2}^{a_{4}-1} \beta^{c_{1}-1} \gamma^{a-1}\right)=\alpha_{1}^{a_{3}-1} \alpha_{2}^{a_{4}-1} \beta^{c_{1}-1} \gamma^{a-1} X_{85}^{(4)}\left(a_{1}, a_{1}, a_{2}, a_{3}, a_{1}, a_{2}, a_{3}, a_{4} c_{1}, c_{2}, c_{3}, c_{1} ; \beta x, y, \alpha, z, u ;\right) \\
& {\left[1-\left(D_{\alpha_{1}} D_{\alpha_{2}} \beta^{-1} D_{\beta}^{-1} \gamma^{-1} D_{\gamma}^{-1} \alpha_{1} \alpha_{2}\right) z\right]^{-a} X_{20}\left(a_{1}, a_{2}, a_{3}, a_{4} ; c_{1}, c_{2} ; x, \alpha_{1} y, \alpha_{2} u\right)} \\
& \times\left(\alpha_{1}^{a_{2}-1} \alpha_{2}^{a_{3}-1} \beta^{c_{3}-1} \gamma^{a-1}\right)=\alpha_{1}^{a_{2}-1} \alpha_{2}^{a_{3}-1} \beta^{c_{3}-1} \gamma^{a-1} X_{85}^{(4)}\left(a_{1}, a_{1}, a_{2}, a_{3}, a_{1}, a_{2}, a_{3}, a_{4} ; c_{1}, c_{2}, c_{3}, c_{1} ; x, \alpha_{1} y, z, \alpha_{2} u\right) .
\end{aligned}
$$

Proof. To prove the result in equality (12) asserted in Theorem 1, let $\varnothing$ denote the left-hand side of equality (12).

Then, employing the series representation of $x_{17}$ and by using (9) and (10), we have

$$
\begin{aligned}
\varnothing & =\sum_{m, n, p, q=0}^{\infty} \frac{\left(a_{1}\right)_{2 m+n}\left(a_{2}\right)_{n+p}\left(a_{3}\right)_{p}(a)_{q} \beta^{-q} \gamma^{-q}}{\left(c_{1}\right)_{m}\left(c_{2}\right)_{n}\left(c_{3}\right)_{p} m ! n ! p ! q !} x^{m} y^{n} z^{p} u^{q} \times D_{\alpha_{1}}^{q} D_{\alpha_{2}}^{q} D_{\beta}^{-q} D_{\gamma}^{-q}\left(\alpha_{1}^{a_{3}+p+q-1} \alpha_{2}^{a_{4}+q-1} \beta^{c+m+q-1} \gamma^{a-1}\right) \\
& =\alpha_{1}^{a_{3}-1} \alpha_{2}^{a_{4}-1} \beta^{c_{1}-1} \gamma^{a-1} \sum_{m, n, p, q=0}^{\infty} \frac{\left(a_{1}\right)_{2 m+n}\left(a_{2}\right)_{n+p}\left(a_{3}\right)_{p+q}\left(a_{4}\right)_{q}}{\left(c_{1}\right)_{m+q}\left(c_{2}\right)_{n}\left(c_{3}\right)_{p}} \frac{(\beta x)^{m}}{m !} \frac{(y)^{n}}{n !} \frac{\left(\alpha_{1} z\right)^{p}}{p !} \frac{u^{q}}{q !}
\end{aligned}
$$




$$
=\alpha_{1}^{a_{3}-1} \alpha_{2}^{a_{4}-1} \beta^{c_{1}-1} \gamma^{a-1} X_{85}^{(4)}\left(a_{1}, a_{1}, a_{2}, a_{3}, a_{1}, a_{2}, a_{3}, a_{4} ; c_{1}, c_{2}, c_{3}, c_{1} ; x, y, z, u\right),
$$

which completes the proof. Similarly, one can prove formulas (13) and (20).
Theorem 2. The following results hold true:

$$
\begin{aligned}
& \quad\left[1-\left(D_{\alpha}^{2} \beta^{-1} D_{\beta}^{-1} \gamma^{-1} D_{\gamma}^{-1} \alpha^{2}\right) x\right]^{-a} F_{P}\left(a_{3}, a_{3}, a_{1}, a_{2}, a_{2}, a_{4} ; c_{3}, c_{1}, c_{1} ; z, \alpha y, u\right) \\
& \quad \times\left(\alpha^{a_{1}-1} \beta^{c_{2}-1} \gamma^{a-1}\right)=\alpha^{a_{1}-1} \beta^{c_{2}-1} \gamma^{a-1} X_{86}^{(4)}\left(a_{1}, a_{1}, a_{2}, a_{3}, a_{1}, a_{2}, a_{3}, a_{4} ; c_{2}, c_{1}, c_{3}, c_{1} ; x, \alpha y, z, u\right), \\
& \quad\left[1-\left(D_{\alpha_{1}} D_{\alpha_{2}} \beta^{-1} D_{\beta}^{-1} \gamma^{-1} D_{\gamma}^{-1} \alpha_{1} \alpha_{2}\right) y\right]_{2}^{-\alpha} F_{1}\left(\frac{a_{1}}{2}, \frac{a_{1}+1}{2} ; c_{2} ; 4 \alpha_{1}^{2} x\right) \\
& \quad \times F_{2}\left(a_{3}, a_{2}, a_{4} ; c_{3}, c_{1} ; \alpha_{2} z, \beta u\right)\left(\alpha_{1}^{a_{1}-1} \alpha_{2}^{a_{2}-1} \beta^{c_{1}-1} \gamma^{a-1}\right)=\alpha_{1}^{a_{1}-1} \alpha_{2}^{a_{2}-1} \beta^{c_{1}-1} \gamma^{a-1} \\
& X_{86}^{(4)}\left(a_{1}, a_{1}, a_{2}, a_{3}, a_{1}, a_{2}, a_{3}, a_{4} ; c_{2}, c_{1}, c_{3}, c_{1} ; \alpha_{1}^{2} x, y, \alpha_{2} z, \beta u\right) .
\end{aligned}
$$

Theorem 3. The following results hold true:

$$
\begin{aligned}
& {\left[1-\left(D_{\alpha_{1}} D_{\alpha_{2}} \beta^{-1} D_{\beta}^{-1} \gamma^{-1} D_{\gamma}^{-1} \alpha_{1} \alpha_{2}\right) u\right]^{-a} X_{16}\left(a_{1}, a_{2}, a_{3} ; c_{1}, c_{2} ; x, \beta y, \alpha_{1} z\right)} \\
& \quad \times\left(\alpha_{1}^{a_{3}-1} \alpha_{2}^{a_{4}-1} \beta^{c_{2}-1} \gamma^{a-1}\right)=\alpha_{1}^{a_{3}-1} \alpha_{2}^{a_{4}-1} \beta^{c_{2}-1} \gamma^{a-1} X_{87}^{(4)}\left(a_{1}, a_{1}, a_{2}, a_{3}, a_{1}, a_{2}, a_{3}, a_{4} ; c_{1}, c_{2}, c_{1}, c_{2} ; x, \beta y, \alpha_{1} z, u\right) \\
& \quad\left[1-\left(D_{\alpha_{1}} D_{\alpha_{2}} \beta^{-1} D_{\beta}^{-1} \gamma^{-1} D_{\gamma}^{-1} \alpha_{1} \alpha_{2}\right) y\right]^{-a} F_{N}\left(a_{4}, \frac{a_{1}}{2}, a_{2}, a_{3}, a_{3}, \frac{a_{1}+1}{2} ; c_{2}, c_{1}, c_{1} ; \beta u, 4 \alpha_{1}^{2} x, \alpha_{2} z\right) \\
& \quad \times\left(\alpha_{1}^{a_{1}-1} \alpha_{2}^{a_{2}-1} \beta^{c_{2}-1} \gamma^{a-1}\right)=\alpha_{1}^{a_{1}-1} \alpha_{2}^{a_{2}-1} \beta^{c_{2}-1} \gamma^{a-1} X_{87}^{(4)}\left(a_{1}, a_{1}, a_{2}, a_{3}, a_{1}, a_{2}, a_{3}, a_{4} ; c_{1}, c_{2}, c_{1}, c_{2} ; \alpha_{1}^{2} x, y, \alpha_{2} z, \beta u\right) .
\end{aligned}
$$

Theorem 4. The following results hold true:

$$
\begin{aligned}
& \quad\left[1-\left(D_{\alpha_{1}} D_{\alpha_{2}} \beta^{-1} D_{\beta}^{-1} \gamma^{-1} D_{\gamma}^{-1} \alpha_{1} \alpha_{2}\right) u\right]^{-a} X_{15}\left(a_{1}, a_{2}, a_{3} ; c_{1}, c_{2} ; \beta x, y, \alpha_{1} z\right) \\
& \quad \times\left(\alpha_{1}^{a_{3}-1} \alpha_{2}^{a_{4}-1} \beta^{c_{1}-1} \gamma^{a-1}\right)=\alpha_{1}^{a_{3}-1} \alpha_{2}^{a_{4}-1} \beta^{c_{1}-1} \gamma^{a-1} X_{88}^{(4)}\left(a_{1}, a_{1}, a_{2}, a_{3}, a_{1}, a_{2}, a_{3}, a_{4} ; c_{1}, c_{2}, c_{2}, c_{1} ; \beta x, y, \alpha_{1} z, u\right), \\
& {\left[1-\left(D_{\alpha}^{2} \beta^{-1} D_{\beta}^{-1} \gamma^{-1} D_{\gamma}^{-1} \alpha^{2}\right) x\right]^{-a} F_{M}\left(a_{4}, a_{2}, a_{2}, a_{3}, a_{3}, a_{1} ; c_{1}, c_{2}, c_{2} ; \beta u, \alpha y, z\right)} \\
& \quad \times\left(\alpha^{a_{1}-1} \beta^{c_{1}-1} \gamma^{a-1}\right)=\alpha^{a_{1}-1} \beta^{c_{1}-1} \gamma^{a-1} X_{88}^{(4)}\left(a_{1}, a_{1}, a_{2}, a_{3}, a_{1}, a_{2}, a_{3}, a_{4} ; c_{1}, c_{2}, c_{2}, c_{1} ; x, \alpha y, z, \beta u\right) .
\end{aligned}
$$

Theorem 5. The following results hold true:

$$
\begin{aligned}
& {\left[1-\left(D_{\alpha_{1}} D_{\alpha_{2}} \beta^{-1} D_{\beta}^{-1} \gamma^{-1} D_{\gamma}^{-1} \alpha_{1} \alpha_{2}\right) u\right]^{-a} X_{16}\left(a_{1}, a_{2}, a_{3} ; c_{1}, c_{2} ; \beta x, y, \alpha_{1} \beta z\right)} \\
& \quad \times\left(\alpha_{1}^{a_{3}-1} \alpha_{2}^{a_{4}-1} \beta^{c_{1}-1} \gamma^{a-1}\right)=\alpha_{1}^{a_{3}-1} \alpha_{2}^{a_{4}-1} \beta^{c_{1}-1} \gamma^{a-1} X_{89}^{(4)}\left(a_{1}, a_{1}, a_{2}, a_{3}, a_{1}, a_{2}, a_{3}, a_{4} ; c_{1}, c_{2}, c_{1}, c_{1} ; \beta x, y, \alpha_{1} \beta z, u\right) \\
& \quad\left[1-\left(D_{\alpha_{1}} D_{\alpha_{2}} \beta^{-1} D_{\beta}^{-1} \gamma^{-1} D_{\gamma}^{-1} \alpha_{1} \alpha_{2}\right) y\right]^{-a} F_{S}\left(\frac{a_{1}}{2}, a_{3}, a_{3}, \frac{a_{1}+1}{2}, a_{2}, a_{4} ; c_{1}, c_{1}, c_{1} ; 4 \alpha_{1} x, \alpha_{2} z, u\right) \\
& \quad \times\left(\alpha_{1}^{a_{1}-1} \alpha_{2}^{a_{2}-1} \beta^{c_{2}-1} \gamma^{a-1}\right)=\alpha_{1}^{a_{1}-1} \alpha_{2}^{a_{2}-1} \beta^{c_{2}-1} \gamma^{a-1} X_{89}^{(4)}\left(a_{1}, a_{1}, a_{2}, a_{3}, a_{1}, a_{2}, a_{3}, a_{4} ; c_{1}, c_{2}, c_{1}, c_{1} ; \alpha_{1} x, y, \alpha_{2} z, u\right)
\end{aligned}
$$


Theorem 6. The following results hold true:

$$
\begin{aligned}
& {\left[1-\left(D_{\alpha_{1}} D_{\alpha_{2}} \beta^{-1} D_{\beta}^{-1} \gamma^{-1} D_{\gamma}^{-1} \alpha_{1} \alpha_{2}\right) z\right]^{-a} X_{18}\left(a_{1}, a_{2}, a_{3}, a_{4} ; c ; \beta x, \alpha_{1} \beta y, \alpha_{2} \beta u\right)} \\
& \times\left(\alpha_{1}^{a_{2}-1} \alpha_{2}^{a_{3}-1} \beta^{c-1} \gamma^{a-1}\right)=\alpha_{1}^{a_{2}-1} \alpha_{2}^{a_{3}-1} \beta^{c-1} \gamma^{a-1} X_{90}^{(4)}\left(a_{1}, a_{1}, a_{2}, a_{3}, a_{1}, a_{2}, a_{3}, a_{4} ; c, c, c, c ; \beta x, \alpha, \beta y, z, \alpha, \beta u\right) \\
& \quad\left[1-\left(D_{\alpha}^{2} \beta^{-1} D_{\beta}^{-1} \gamma^{-1} D_{\gamma}^{-1} \alpha^{2}\right) x\right]^{-a} F_{T}\left(a_{4}, a_{2}, a_{2}, a_{3}, a_{1}, a_{3} ; c, c, c ; \beta u, \alpha \beta y, \beta z\right) \\
& \quad \times\left(\alpha^{a_{1}-1} \beta^{c-1} \gamma^{a-1}\right)=\alpha^{a_{1}-1} \beta^{c-1} \gamma^{a-1} X_{90}^{(4)}\left(a_{1}, a_{1}, a_{2}, a_{3}, a_{1}, a_{2}, a_{3}, a_{4} c, c, c, c ; x, \alpha \beta y, \beta z, \beta u ;\right)
\end{aligned}
$$

\section{Differentiation Formulas}

Theorem 7. The following derivative formulas hold true:

The results of this section can be derived from formula (9) by a direct evaluation.

$$
\begin{aligned}
& D_{w_{1}}^{a_{1}-c} D_{w_{2}}^{a_{2}-c^{\prime}}\left[w_{1}^{a_{1}-1} w_{2}^{a_{2}-1} X_{85}^{(4)}\left(c, c, c^{\prime}, a_{3}, c, c^{\prime}, a_{3}, a_{4} ; c_{1}, c_{2}, c_{3}, c_{1} ; w_{1}^{2} x, w_{1} w_{2} y, w_{2} z, u\right)\right] \\
& \quad=\frac{\Gamma\left(a_{1}\right) \Gamma\left(a_{2}\right)}{\Gamma(c) \Gamma\left(c^{\prime}\right)} w_{1}^{c-1} w_{2}^{c^{\prime}-1} X_{85}^{(4)}\left(a_{1}, a_{1}, a_{2}, a_{3}, a_{1}, a_{2}, a_{3}, a_{4} ; c_{1}, c_{2}, c_{3}, c_{1} ; w_{1}^{2} x, w_{1} w_{2} y, w_{2} z, u\right), \\
& D_{w_{1}}^{a_{2}-c} D_{w_{2}}^{a_{3}-c^{\prime}}\left[w_{1}^{a_{2}-1} w_{2}^{a_{3}-1} X_{85}^{(4)}\left(a_{1}, a_{1}, c, c^{\prime}, a_{1}, c, c^{\prime}, a_{4} ; c_{1}, c_{2}, c_{3}, c_{1} ; x, w_{1} y, w_{1} w_{2} z, w_{2} u\right)\right] \\
& \quad=\frac{\Gamma\left(a_{2}\right) \Gamma\left(a_{3}\right)}{\Gamma(c) \Gamma\left(c^{\prime}\right)} w_{1}^{c-1} w_{2}^{c^{\prime}-1} X_{85}^{(4)}\left(a_{1}, a_{1}, a_{2}, a_{3}, a_{1}, a_{2}, a_{3}, a_{4} ; c_{1}, c_{2}, c_{3}, c_{1} ; x, w_{1} y, w_{1} w_{2} z, w_{2} u\right), \\
& D_{w_{1}}^{a_{3}-c} D_{w_{2}}^{a_{4}-c^{\prime}}\left[w_{1}^{a_{3}-1} w_{2}^{a_{4}-1} X_{85}^{(4)}\left(a_{1}, a_{1}, a_{2}, c, a_{1}, a_{2}, c, c^{\prime} ; c_{1}, c_{2}, c_{3}, c_{1} ; x, y, w_{1} z, w_{1} w_{2} u\right)\right] \\
& \quad=\frac{\Gamma\left(a_{3}\right) \Gamma\left(a_{4}\right)}{\Gamma(c) \Gamma\left(c^{\prime}\right)} w_{1}^{c-1} w_{2}^{c^{\prime}-1} X_{85}^{(4)}\left(a_{1}, a_{1}, a_{2}, a_{3}, a_{1}, a_{2}, a_{3}, a_{4} ; c_{1}, c_{2}, c_{3}, c_{1} ; x, y, w_{1} z, w_{1} w_{2} u\right) .
\end{aligned}
$$

Theorem 8. The following differentiation formulas hold:

$$
\begin{aligned}
& D_{x}^{a_{1}-c}\left[x^{a_{1}-1} X_{86}^{(4)}\left(c, c, a_{2}, a_{3}, c, a_{2}, a_{3}, a_{4} ; c_{2}, c_{1}, c_{3}, c_{1} ; x^{2}, x y, z, u\right)\right] \\
& \quad=\frac{\Gamma\left(a_{1}\right)}{\Gamma(c)} x^{c-1} X_{86}^{(4)}\left(a_{1}, a_{1}, a_{2}, a_{3}, a_{1}, a_{2}, a_{3}, a_{4} ; c_{2}, c_{1}, c_{3}, c_{1} ; x^{2}, x y, z, u\right), \\
& D_{x}^{a_{1}-c} D_{y}^{a_{2}-c^{\prime}}\left[x^{a_{1}-1} y^{a_{2}-1} X_{86}^{(4)}\left(c, c, c^{\prime}, a_{3}, c, c^{\prime}, a_{3}, a_{4} ; c_{2}, c_{1}, c_{3}, c_{1} ; x^{2}, x y, y z, u\right)\right] \\
& \quad=\frac{\Gamma\left(a_{1}\right) \Gamma\left(a_{2}\right)}{\Gamma(c) \Gamma\left(c^{\prime}\right)} x^{c-1} y^{c^{\prime}-1} X_{86}^{(4)}\left(a_{1}, a_{1}, a_{2}, a_{3}, a_{1}, a_{2}, a_{3}, a_{4} ; c_{2}, c_{1}, c_{3}, c_{1} ; x^{2}, x y, y z, u\right), \\
& D_{u}^{a_{4}-c}\left[u^{a_{4}-1} X_{86}^{(4)}\left(a_{1}, a_{1}, a_{2}, a_{3}, a_{1}, a_{2}, a_{3}, c ; c_{2}, c_{1}, c_{3}, c_{1} ; x, y, z, u\right)\right] \\
& =\frac{\Gamma\left(a_{4}\right)}{\Gamma(c)} u^{c-1} X_{86}^{(4)}\left(a_{1}, a_{1}, a_{2}, a_{3}, a_{1}, a_{2}, a_{3}, a_{4} ; c_{2}, c_{1}, c_{3}, c_{1} ; x, y, z, u\right) .
\end{aligned}
$$


Theorem 9. The following derivative formulas hold true:

$$
\begin{aligned}
& D_{w}^{a_{2}-c} D_{z}^{a_{3}-c^{\prime}}\left[w^{a_{2}-1} z^{a_{3}-1} X_{87}^{(4)}\left(a_{1}, a_{1}, c, c^{\prime}, a_{1}, c, c^{\prime}, a_{4} ; c_{1}, c_{2}, c_{1}, c_{2} ; x, w y, w z, u z\right)\right] \\
& \quad=\frac{\Gamma\left(a_{2}\right) \Gamma\left(a_{3}\right)}{\Gamma(c) \Gamma\left(c^{\prime}\right)} w^{c-1} z^{c^{\prime}-1} X_{87}^{(4)}\left(a_{1}, a_{1}, a_{2}, a_{3}, a_{1}, a_{2}, a_{3}, a_{4} ; c_{1}, c_{2}, c_{1}, c_{2} ; x, w y, w z, u z\right) \\
& D_{w}^{a_{1}-c} D_{y}^{a_{2}-c^{\prime}} D_{z}^{a_{3}-c^{\prime \prime}}\left[w^{a_{1}-1} y^{a_{2}-1} z^{a_{3}-1} X_{87}^{(4)}\left(c, c, c^{\prime}, c^{\prime \prime}, c, c^{\prime}, c^{\prime \prime}, a_{4} ; c_{1}, c_{2}, c_{1}, c_{2} ; w^{2} x, w y, y z, u z\right)\right] \\
& \quad=\frac{\Gamma\left(a_{1}\right) \Gamma\left(a_{2}\right) \Gamma\left(a_{3}\right)}{\Gamma(c) \Gamma\left(c^{\prime}\right) \Gamma\left(c^{\prime \prime}\right)} w^{c-1} y^{c^{\prime}-1} z^{c^{\prime \prime}-1} X_{87}^{(4)}\left(a_{1}, a_{1}, a_{2}, a_{3}, a_{1}, a_{2}, a_{3}, a_{4} ; c_{1}, c_{2}, c_{1}, c_{2} ; w^{2} x, w y, y z, u z\right) \\
& D_{w_{1}}^{a_{1}-c} D_{w_{2}}^{a_{4}-c^{\prime}}\left[w_{1}^{a_{1}-1} w_{2}^{a_{4}-1} X_{87}^{(4)}\left(c, c, a_{2}, a_{3}, c, a_{2}, a_{3} c^{\prime} ; c_{1}, c_{2}, c_{1}, c_{2} ; w_{1}^{2} x, w_{1} y, z, w_{2} u\right)\right] \\
& \quad=\frac{\Gamma\left(a_{1}\right) \Gamma\left(a_{4}\right)}{\Gamma(c) \Gamma\left(c^{\prime}\right)} w_{1}^{c-1} w_{2}^{c^{\prime}-1} X_{87}^{(4)}\left(a_{1}, a_{1}, a_{2}, a_{3}, a_{1}, a_{2}, a_{3}, a_{4} ; c_{1}, c_{2}, c_{1}, c_{2} ; w_{1}^{2} x, w_{1} y, z, w_{2} u\right)
\end{aligned}
$$

Theorem 10. The following derivative formulas hold true:

$$
\begin{aligned}
& D_{y}^{a_{2}-c} {\left[y^{a_{2}-1} X_{88}^{(4)}\left(a_{1}, a_{1}, c, a_{3}, a_{1}, c, a_{3}, a_{4} ; c_{1}, c_{2}, c_{2}, c_{1} ; x, y, y z, u\right)\right] } \\
&= \frac{\Gamma\left(a_{2}\right)}{\Gamma(c)} y^{c-1} X_{88}^{(4)}\left(a_{1}, a_{1}, a_{2}, a_{3}, a_{1}, a_{2}, a_{3}, a_{4} ; c_{1}, c_{2}, c_{2}, c_{1} ; x, y, y z, u\right), \\
& D_{z}^{a_{2}-c}\left[z^{a_{2}-1} X_{88}^{(4)}\left(a_{1}, a_{1}, c, a_{3}, a_{1}, c, a_{3}, a_{4} ; c_{1}, c_{2}, c_{2}, c_{1} ; x, y z, z, u\right)\right] \\
&=\frac{\Gamma\left(a_{2}\right)}{\Gamma(c)} z^{c-1} X_{88}^{(4)}\left(a_{1}, a_{1}, a_{2}, a_{3}, a_{1}, a_{2}, a_{3}, a_{4} ; c_{1}, c_{2}, c_{2}, c_{1} ; x, y z, z, u\right), \\
& D_{w_{1}}^{a_{1}-c} D_{w_{2}}^{a_{2}-c^{\prime}} D_{w_{3}}^{a_{3}-c^{\prime \prime}} D_{w_{4}}^{a_{4}-c^{\prime \prime \prime}}\left[w_{1}^{a_{1}-1} w_{2}^{a_{2}-1} w_{3}^{a_{3}-1} w_{4}^{a_{4}-1} X_{88}^{(4)}\left(c, c, c^{\prime}, c^{\prime \prime}, c, c^{\prime}, c^{\prime \prime}, c^{\prime \prime \prime} ; c_{1}, c_{2}, c_{2}, c_{1} ; w_{1}^{2} x, w_{1} w_{2} y, w_{2} w_{3} z, w_{3} w_{4} u\right)\right] \\
&=\frac{\Gamma\left(a_{1}\right) \Gamma\left(a_{2}\right) \Gamma\left(a_{3}\right) \Gamma\left(a_{4}\right)}{\Gamma(c) \Gamma\left(c^{\prime}\right) \Gamma\left(c^{\prime \prime}\right) \Gamma\left(c^{\prime \prime \prime}\right)} w_{1}^{c-1} w_{2}^{c^{\prime}-1} w_{3}^{c^{\prime \prime}-1} w_{4}^{c^{\prime \prime \prime}-1} \\
& \quad \times X_{88}^{(4)}\left(a_{1}, a_{1}, a_{2}, a_{3}, a_{1}, a_{2}, a_{3}, a_{4} ; c_{1}, c_{2}, c_{2}, c_{1} ; w_{1}^{2} x, w_{1} w_{2} y, w_{2} w_{3} z, w_{3} w_{4} u\right) .
\end{aligned}
$$

Theorem 11. The following derivative formulas hold true:

$$
\begin{aligned}
& D_{y}^{a_{1}-c}\left[y^{a_{1}-1} X_{89}^{(4)}\left(c, c, a_{2}, a_{3}, c, a_{2}, a_{3}, a_{4} ; c_{1}, c_{2}, c_{1}, c_{1} ; x y^{2}, y, z, u\right)\right] \\
& \quad=\frac{\Gamma\left(a_{1}\right)}{\Gamma(c)} y^{c-1} X_{88}^{(4)}\left(a_{1}, a_{1}, a_{2}, a_{3}, a_{1}, a_{2}, a_{3}, a_{4} ; c_{1}, c_{2}, c_{1}, c_{1} ; x y^{2}, y, z, u\right) \\
& D_{y}^{a_{1}-c} D_{w}^{a_{2}-c^{\prime}}\left[y^{a_{1}-1} w^{a_{2}-1} X_{89}^{(4)}\left(c, c, c^{\prime}, a_{3}, c, c^{\prime}, a_{3}, a_{4} ; c_{1}, c_{2}, c_{1}, c_{1} ; x y^{2}, w y, w z, u\right)\right]
\end{aligned}
$$




$$
\begin{aligned}
&=\frac{\Gamma\left(a_{1}\right) \Gamma\left(a_{2}\right)}{\Gamma(c) \Gamma\left(c^{\prime}\right)} y^{c-1} w^{c^{\prime}-1} X_{89}^{(4)}\left(a_{1}, a_{1}, a_{2}, a_{3}, a_{1}, a_{2}, a_{3}, a_{4} ; c_{1}, c_{2}, c_{1}, c_{1} ; x y^{2}, w y, w z, u\right), \\
& D_{x}^{a_{1}-c} D_{w_{1}}^{a_{2}-c^{\prime \prime}} D_{z}^{a_{3}-c^{\prime \prime}} D_{w_{2}}^{a_{4}-c^{\prime \prime \prime}}\left[x^{a_{1}-1} w_{1}^{a_{2}-1} z^{a_{3}-1} w_{2}^{a_{4}-1} X_{89}^{(4)}\left(c, c, c^{\prime}, c^{\prime \prime}, c, c^{\prime}, c^{\prime \prime}, c^{\prime \prime} ; c_{1}, c_{2}, c_{1}, c_{1} ; x^{2}, w_{1} x y, w_{1} z, w_{2} u z\right)\right] \\
&=\frac{\Gamma\left(a_{1}\right) \Gamma\left(a_{2}\right) \Gamma\left(a_{3}\right) \Gamma\left(a_{4}\right)}{\Gamma(c) \Gamma\left(c^{\prime}\right) \Gamma\left(c^{\prime \prime}\right) \Gamma\left(c^{\prime \prime \prime}\right)} x^{c-1} w_{1}^{c^{\prime}-1} z^{c^{\prime \prime}-1} w_{2}^{c^{\prime \prime}-1} \times X_{89}^{(4)}\left(a_{1}, a_{1}, a_{2}, a_{3}, a_{1}, a_{2}, a_{3}, a_{4} ; c_{1}, c_{2}, c_{1}, c_{1} ; x^{2}, w_{1} x y, w_{1} z, w_{2} u z\right) .
\end{aligned}
$$

Theorem 12. The following derivative formulas hold true:

$$
\begin{aligned}
& D_{z}^{a_{2}-c^{\prime}} D_{u}^{a_{3}-c^{\prime \prime}}\left[z^{a_{2}-1} u^{a_{3}-1} X_{90}^{(4)}\left(a_{1}, a_{1}, c^{\prime}, c^{\prime \prime}, a_{1}, c^{\prime}, c^{\prime \prime}, a_{4} ; c, c, c, c ; x, y z, u z, u\right)\right] \\
& =\frac{\Gamma\left(a_{2}\right) \Gamma\left(a_{3}\right)}{\Gamma(c) \Gamma\left(c^{\prime}\right)} z^{c^{\prime}-1} u^{c^{\prime \prime}-1} X_{90}^{(4)}\left(a_{1}, a_{1}, a_{2}, a_{3}, a_{1}, a_{2}, a_{3}, a_{4} ; c, c, c, c ; x, y z, u z, u\right), \\
& D_{y}^{a_{2}-c^{\prime}} D_{w}^{a_{3}-c^{\prime \prime}} D_{u}^{a_{4}-c^{\prime \prime \prime}}\left[y^{a_{2}-1} w^{a_{3}-1} u^{a_{4}-1} X_{90}^{(4)}\left(a_{1}, a_{1}, c^{\prime}, c^{\prime \prime}, a_{1}, c^{\prime}, c^{\prime \prime}, c^{\prime \prime \prime} ; c, c, c, c ; x, y, w y z, w u\right)\right] \\
& =\frac{\Gamma\left(a_{2}\right) \Gamma\left(a_{3}\right) \Gamma\left(a_{4}\right)}{\Gamma\left(c^{\prime}\right) \Gamma\left(c^{\prime \prime}\right) \Gamma\left(c^{\prime \prime \prime}\right)} y^{c^{\prime}-1} w^{c^{\prime \prime}-1} u^{c^{\prime \prime \prime}-1} X_{90}^{(4)}\left(a_{1}, a_{1}, a_{2}, a_{3}, a_{1}, a_{2}, a_{3}, a_{4} ; c, c, c, c ; x, y, w y z, w u\right), \\
& D_{w_{1}}^{a_{1}-c^{\prime}} D_{z}^{a_{3}-c^{\prime \prime}} D_{w_{2}}^{a_{4}-c^{\prime \prime \prime}}\left[w_{1}^{a_{1}-1} z^{a_{3}-1} w_{2}^{a_{4}-1} X_{90}^{(4)}\left(c^{\prime}, c^{\prime}, a_{2}, c^{\prime \prime}, c^{\prime}, a_{2}, c^{\prime \prime}, c ; c, c, c, c ; w_{1}^{2} x, w_{1} y, z, w_{2} u z\right)\right] \\
& =\frac{\Gamma\left(a_{1}\right) \Gamma\left(a_{3}\right) \Gamma\left(a_{4}\right)}{\Gamma\left(c^{\prime}\right) \Gamma\left(c^{\prime \prime}\right) \Gamma\left(c^{\prime \prime \prime}\right)} w_{2}^{c-1} z^{c^{\prime \prime}-1} w_{2}^{c^{\prime \prime \prime}-1} X_{90}^{(4)}\left(a_{1}, a_{1}, a_{2}, a_{3}, a_{1}, a_{2}, a_{3}, a_{4} ; c, c, c, c ; w_{1}^{2} x, w_{1} y, z, w_{2} u z\right) .
\end{aligned}
$$

\section{Integral Representations}

In this section, we give integral representations of Laplace type for our new hypergeometric functions of four variables.
Theorem 13. Each of the following integral representations holds true:

$$
\begin{aligned}
& X_{85}^{(4)}\left(a_{1}, a_{1}, a_{2}, a_{3}, a_{1}, a_{2}, a_{3}, a_{4} ; c_{1}, c_{2}, c_{3}, c_{1} ; x, y, z, u\right) \\
& \quad=\frac{1}{\Gamma\left(a_{1}\right) \Gamma\left(a_{3}\right)} \int_{0}^{\infty} \int_{0}^{\infty} x e^{-(s+t)} s^{a_{1}-1} t^{a_{3}-1} \Phi_{3}\left(a_{4} ; c_{1} ; t u, s^{2} x\right) \Psi_{2}\left(a_{4} ; c_{2}, c_{3} ; s y, t z\right) \mathrm{d} s \mathrm{~d} t\left(\operatorname{Re}\left(a_{1}\right)>0, \operatorname{Re}\left(a_{3}\right)>0\right) \\
& X_{86}^{(4)}\left(a_{1}, a_{1}, a_{2}, a_{3}, a_{1}, a_{2}, a_{3}, a_{4} ; c_{2}, c_{1}, c_{3}, c_{1} ; x, y, z, u\right)=\frac{1}{\Gamma\left(a_{1}\right) \Gamma\left(a_{2}\right) \Gamma\left(a_{3}\right)} \\
& \quad \cdot \int_{0}^{\infty} \int_{0}^{\infty} \int_{0}^{\infty} \times e^{-(s+t+v)} s^{a_{1}-1} t^{a_{2}-1} v^{a_{3}-1} \Phi_{3}\left(a_{4} ; c_{1} ; v u, s t y\right)_{0} F_{1}\left(-; c_{2} ; s^{2} x\right)_{0} F_{1}\left(-; c_{3} ; t v z\right) \mathrm{d} s \mathrm{~d} t \mathrm{~d} v \\
& \quad \cdot\left(\operatorname{Re}\left(a_{1}\right)>0, \operatorname{Re}\left(a_{2}\right)>0, \operatorname{Re}\left(a_{3}\right)>0\right), \\
& X_{87}^{(4)}\left(a_{1}, a_{1}, a_{2}, a_{3}, a_{1}, a_{2}, a_{3}, a_{4} ; c_{1}, c_{2}, c_{1}, c_{2} ; x, y, z, u\right)=\frac{1}{\Gamma\left(a_{1}\right) \Gamma\left(a_{2}\right) \Gamma\left(a_{3}\right)} \\
& \quad \cdot \int_{0}^{\infty} \int_{0}^{\infty} \int_{0}^{\infty} \times e^{-(s+t+v)} s^{a_{1}-1} t^{a_{2}-1} v^{a_{3}-1}{ }_{0} F_{1}\left(-; c_{1} ; s^{2} x+t v z\right) \Phi_{3}\left(a_{4} ; c_{2} ; v u, s t y\right) \mathrm{d} s \mathrm{~d} t \mathrm{~d} v \\
& \quad \cdot\left(\operatorname{Re}\left(a_{1}\right)>0, \operatorname{Re}\left(a_{2}\right)>0, \operatorname{Re}\left(a_{3}\right)>0\right),
\end{aligned}
$$




$$
\begin{gathered}
X_{88}^{(4)}\left(a_{1}, a_{1}, a_{2}, a_{3}, a_{1}, a_{2}, a_{3}, a_{4} ; c_{1}, c_{2}, c_{2}, c_{1} ; x, y, z, u\right)=\frac{1}{\Gamma\left(a_{1}\right) \Gamma\left(a_{3}\right)} \\
\cdot \int_{0}^{\infty} \int_{0}^{\infty} x e^{-(s+t)} s^{a_{1}-1} t^{a_{3}-1} \Phi_{3}\left(a_{4} ; c_{1} ; t u, s^{2} x\right)_{1} F_{1}\left(a_{2} ; c_{2} ; s y+t z\right) \mathrm{d} s \mathrm{~d} t\left(\operatorname{Re}\left(a_{1}\right)>0, \operatorname{Re}\left(a_{3}\right)>0\right), \\
X_{89}^{(4)}\left(a_{1}, a_{1}, a_{2}, a_{3}, a_{1}, a_{2}, a_{3}, a_{4} ; c_{1}, c_{2}, c_{1}, c_{1} ; x, y, z, u\right)=\frac{1}{\Gamma\left(a_{1}\right) \Gamma\left(a_{2}\right) \Gamma\left(a_{4}\right)} \\
\cdot \int_{0}^{\infty} \int_{0}^{\infty} \int_{0}^{\infty} \times e^{-(s+t+v)} s^{a_{1}-1} t^{a_{2}-1} v^{a_{4}-1} \Phi_{3}\left(a_{3} ; c_{1} ; t z+v u, s^{2} x\right)_{0} F_{1}\left(-; c_{2} ; s t y\right) \mathrm{d} s \mathrm{~d} t \mathrm{~d} v\left(\operatorname{Re}\left(a_{1}\right)>0, \operatorname{Re}\left(a_{2}\right)>0, \operatorname{Re}\left(a_{4}\right)>0\right), \\
\quad X_{90}^{(4)}\left(a_{1}, a_{1}, a_{2}, a_{3}, a_{1}, a_{2}, a_{3}, a_{4} ; c, c, c, c ; x, y, z, u\right)=\frac{1}{\Gamma\left(a_{1}\right) \Gamma\left(a_{3}\right)} \quad(31) \\
\cdot \int_{0}^{\infty} \int_{0}^{\infty} \times e^{-(s+t)} s^{a_{1}-1} t^{a_{3}-1} \Phi_{3}^{(3)}\left(a_{2}, a_{4} ; c ; s y+t z, t u, s^{2} x\right) \mathrm{d} s \mathrm{~d} t\left(\operatorname{Re}\left(a_{1}\right)>0, \operatorname{Re}\left(a_{3}\right)>0\right), \\
\text { where }{ }_{0} F_{1}, F_{1}, \Psi_{2}, \Phi_{3}, \text { and } \Phi_{3}^{(3)} \text { are the confluent hyper- } \quad \Gamma(z)=\int_{0}^{\infty} e^{-t} t^{z-1} \mathrm{~d} t, \quad(\Re(z)>0) .
\end{gathered}
$$
geometric functions defined by (see [11])

$$
\begin{aligned}
{ }_{0} F_{1}(-; c ; x) & =\sum_{m=0}^{\infty} \frac{1}{(c)_{m}} \frac{x^{m}}{m !}, \\
{ }_{1} F_{1}(a ; c ; x) & =\sum_{m=0}^{\infty} \frac{(a)_{m}}{(c)_{m}} \frac{x^{m}}{m !}, \\
\Psi_{2}(a ; b, c ; x, y) & =\sum_{m=0}^{\infty} \frac{(a)_{m+n}}{(b)_{m}(c)_{n}} \frac{x^{m}}{m !} \frac{y^{n}}{n !}, \\
\Phi_{3}(a ; c ; x, y) & =\sum_{m, n=0}^{\infty} \frac{(a)_{m}}{(c)_{m+n}} \frac{x^{m}}{m !} \frac{y^{n}}{n !}, \\
\Phi_{3}^{(3)}(a, b ; c ; x, y, z) & =\sum_{m, n=0}^{\infty} \frac{(a)_{m}(b)_{n}}{(c)_{m+n+p}} \frac{x^{m}}{m !} \frac{y^{n}}{n !} \frac{z^{p}}{p !} .
\end{aligned}
$$

Proof. It is noted that each of the integral representations (27) to (32) can be proved mainly by expressing the series definition of the involved special functions in each integrand, changing the order of the integral sign and the summation, and finally using the following well-known integral formula $[12,17]$ :

\section{Operator Formulas}

Here, we establish some operator identities for functions $X_{85}^{(4)}, X_{86}^{(4)}, \ldots, X_{90}^{(4)}$. We begin by recalling the following reciprocally inverse operators (see $[3,18])$ :

$$
\begin{aligned}
H_{t_{1}, \ldots, t_{i}}(a, b) & :=\frac{\Gamma(b) \Gamma\left(a+\delta_{1}+\cdots+\delta_{i}\right)}{\Gamma(a) \Gamma\left(b+\delta_{1}+\cdots+\delta_{i}\right)} \\
& =\sum_{k_{1}, \ldots, k_{i}=0}^{\infty} \frac{(b-a)_{k_{1}+\cdots+k_{i}}\left(-\delta_{1}\right)_{k_{1}} \ldots\left(-\delta_{i}\right)_{k_{i}}}{(b)_{k_{1}+\cdots+k_{i}} k_{1} ! \ldots k_{i} !} \\
\bar{H}_{t_{1}, \ldots, t_{i}}(a, b) & :=\frac{\Gamma(a) \Gamma\left(a+\delta_{1}+\cdots+\delta_{i}\right)}{\Gamma(b) \Gamma\left(a+\delta_{1}+\cdots+\delta_{i}\right)} \\
& =\sum_{k_{1}, \ldots, k_{i}=0}^{\infty} \frac{(b-a)_{k_{1}+\cdots+k_{i}}\left(-\delta_{1}\right)_{k_{1}} \ldots\left(-\delta_{i}\right)_{k_{i}}}{\left(1-a-\delta_{1}-\cdots-\delta_{i}\right)_{k_{1}+\cdots+k_{i}} k_{1} ! \ldots k_{i} !},
\end{aligned}
$$

where $\delta_{j}:=t_{j}\left(\partial / \partial t_{j}\right), j=1, \ldots, i ; i \in \mathbb{N}:=\{1,2,3, \ldots\}$.

Theorem 14. The following identities hold true:

$$
\begin{aligned}
& X_{85}^{(4)}\left(a_{1}, a_{1}, a_{2}, a_{3}, a_{1}, a_{2}, a_{3}, a_{4} ; c_{1}, c_{2}, c_{3}, c_{1} ; x, y, z, u\right)=H_{y, z}\left(a_{2}, a\right) X_{85}^{(4)}\left(a_{1}, a_{1}, a, a_{3}, a_{1}, a, a_{3}, a_{4} ; c_{1}, c_{2}, c_{3}, c_{1} ; x, y, z, u\right), \\
& X_{85}^{(4)}\left(a_{1}, a_{1}, a_{2}, a_{3}, a_{1}, a_{2}, a_{3}, a_{4} ; c_{1}, c_{2}, c_{3}, c_{1} ; x, y, z, u\right)=H_{z}\left(c, c_{3}\right) X_{85}^{(4)}\left(a_{1}, a_{1}, a_{2}, a_{3}, a_{1}, a_{2}, a_{3}, a_{4} ; c_{1}, c_{2}, c, c_{1} ; x, y, z, u\right) .
\end{aligned}
$$


Theorem 15. The following identities hold true:

$$
\begin{aligned}
& X_{86}^{(4)}\left(a_{1}, a_{1}, a_{2}, a_{3}, a_{1}, a_{2}, a_{3}, a_{4} ; c_{2}, c_{1}, c_{3}, c_{1} ; x, y, z, u\right)=\bar{H}_{z, u}\left(a, a_{3}\right) X_{86}^{(4)}\left(a_{1}, a_{1}, a_{2}, a, a_{1}, a_{2}, a, a_{4} ; c_{2}, c_{1}, c_{3}, c_{1} ; x, y, z, u\right), \\
& X_{86}^{(4)}\left(a_{1}, a_{1}, a_{2}, a_{3}, a_{1}, a_{2}, a_{3}, a_{4} ; c_{2}, c_{1}, c_{3}, c_{1} ; x, y, z, u\right)=\bar{H}_{x}\left(c_{2}, c\right) X_{86}^{(4)}\left(a_{1}, a_{1}, a_{2}, a_{3}, a_{1}, a_{2}, a_{3}, a_{4} ; c, c_{1}, c_{3}, c_{1} ; x, y, z, u\right) .
\end{aligned}
$$

Theorem 16. The following identities hold true:

$$
\begin{aligned}
& X_{87}^{(4)}\left(a_{1}, a_{1}, a_{2}, a_{3}, a_{1}, a_{2}, a_{3}, a_{4} ; c_{1}, c_{2}, c_{1}, c_{2} ; x, y, z, u\right)=H_{u}\left(a_{4}, a\right) X_{87}^{(4)}\left(a_{1}, a_{1}, a_{2}, a_{3}, a_{1}, a_{2}, a_{3}, a ; c_{1}, c_{2}, c_{1}, c_{2} ; x, y, z, u\right) \\
& X_{87}^{(4)}\left(a_{1}, a_{1}, a_{2}, a_{3}, a_{1}, a_{2}, a_{3}, a_{4} ; c_{1}, c_{2}, c_{1}, c_{2} ; x, y, z, u\right)=H_{x, z}\left(c, c_{1}\right) H_{y, u}\left(c^{\prime}, c_{2}\right) X_{87}^{(4)}\left(a_{1}, a_{1}, a_{2}, a_{3}, a_{1}, a_{2}, a_{3}, a_{4} ; c, c^{\prime}, c, c^{\prime} ; x, y, z, u\right)
\end{aligned}
$$

Theorem 17. The following identities hold true:

$$
X_{88}^{(4)}\left(a_{1}, a_{1}, a_{2}, a_{3}, a_{1}, a_{2}, a_{3}, a_{4} ; c_{1}, c_{2}, c_{2}, c_{1} ; x, y, z, u\right)=\bar{H}_{y, z}\left(a, a_{2}\right) \bar{H}_{u}\left(a^{\prime}, a_{4}\right) X_{88}^{(4)}\left(a_{1}, a_{1}, a, a_{3}, a_{1}, a, a_{3}, a^{\prime} ; c_{1}, c_{2}, c_{2}, c_{1} ; x, y, z, u\right)
$$

$X_{88}^{(4)}\left(a_{1}, a_{1}, a_{2}, a_{3}, a_{1}, a_{2}, a_{3}, a_{4} ; c_{1}, c_{2}, c_{2}, c_{1} ; x, y, z, u\right)=H_{x, u}\left(c, c_{1}\right) X_{88}^{(4)}\left(a_{1}, a_{1}, a_{2}, a_{3}, a_{1}, a_{2}, a_{3}, a_{4} ; c, c_{2}, c_{2}, c ; x, y, z, u\right)$

Theorem 18. The following identities hold true:

$$
\begin{aligned}
& X_{89}^{(4)}\left(a_{1}, a_{1}, a_{2}, a_{3}, a_{1}, a_{2}, a_{3}, a_{4} ; c_{1}, c_{2}, c_{1}, c_{1} ; x, y, z, u\right)=H_{x, z, u}\left(c, c_{1}\right) X_{89}^{(4)}\left(a_{1}, a_{1}, a_{2}, a_{3}, a_{1}, a_{2}, a_{3}, a_{4} ; c, c, c, c ; x, y, z, u\right) \\
& X_{89}^{(4)}\left(a_{1}, a_{1}, a_{2}, a_{3}, a_{1}, a_{2}, a_{3}, a_{4} ; c_{1}, c_{2}, c_{1}, c_{1} ; x, y, z, u\right)=\bar{H}_{x, z, u}\left(c_{1}, c\right) X_{89}^{(4)}\left(a_{1}, a_{1}, a_{2}, a_{3}, a_{1}, a_{2}, a_{3}, a_{4} ; c, c_{2}, c, c ; x, y, z, u\right) .
\end{aligned}
$$

Theorem 19. The following identities hold true:

$$
\begin{aligned}
& X_{90}^{(4)}\left(a_{1}, a_{1}, a_{2}, a_{3}, a_{1}, a_{2}, a_{3}, a_{4} ; c, c, c, c ; x, y, z, u\right)=\bar{H}_{u}\left(a, a_{4}\right) X_{90}^{(4)}\left(a_{1}, a_{1}, a_{2}, a_{3}, a_{1}, a_{2}, a_{3}, a ; c, c, c, c ; x, y, z, u\right), \\
& X_{90}^{(4)}\left(a_{1}, a_{1}, a_{2}, a_{3}, a_{1}, a_{2}, a_{3}, a_{4} ; c, c, c, c ; x, y, z, u\right)=H_{z, u}\left(a_{3}, a\right) X_{90}^{(4)}\left(a_{1}, a_{1}, a_{2}, a, a_{1}, a_{2}, a, a_{4} ; c, c, c, c ; x, y, z, u\right) .
\end{aligned}
$$

Proof. Relations (37) to (47) can be proved by means of Mellin and Mellin-Barnes integral representation methods for hypergeometric functions (see [19]). The details of proofs are omitted.

\section{Data Availability}

The data used to support the findings of this study are available from the corresponding author upon request. 


\section{Conflicts of Interest}

The authors declare that they have no conflicts of interest.

\section{Authors' Contributions}

All authors contributed equally and significantly to writing this article. All authors read and approved the final manuscript.

\section{References}

[1] M. G. Bin-Saad, K. S. Nisar, and J. A. Younis, Fractional Differentiations and Integrations of Quadruple Hypergeometric Series, Communications of the Korean Mathematical Society, Seoul, South Korea, 2020, in Press.

[2] Y. A. Brychkov and N. Saad, "On some formulas for the Appell function $\mathrm{F}_{2}\left(\mathrm{a}, \mathrm{b}, \mathrm{b}^{\prime} ; \mathrm{c}, \mathrm{c}^{\prime} ; \mathrm{w} ; \mathrm{z}\right)$," Integral Transforms and Special Functions, vol. 25, no. 2, pp. 111-123, 2014.

[3] A. Hasanov and J. A. Younis, " $H(\alpha, \beta) \bar{H}(\alpha, \beta)$ Application of operators and to hypergeometric Gaussian functions in three variables," Acta Universitatis Apulensis, vol. 64, pp. 127-136, 2020.

[4] A. Hasanov and J. A. Younis, "Decomposition formulas for some Gaussian hypergeometric functions in three variables," East-West Journal of Mathematics, vol. 22, pp. 133-140, 2020.

[5] S. B. Opps, N. Saad, and H. M. Srivastava, "Recursion formulas for Appell's hypergeometric function with some applications to radiation field problems," Applied Mathematics and Computation, vol. 207, no. 2, pp. 545-558, 2009.

[6] V. Sahai and A. Verma, "Recursion formulas for the Srivastava-Daoust and related multivariable hypergeometric functions," Asian-European Journal of Mathematics, vol. 9, p. 4, 2016.

[7] H. Exton, Multiple Hypergeometric Functions and Applications, Halsted Press, New York, NY, USA, 1976.

[8] C. Sharma and C. L. Parihar, "Hypergeometric functions of four variables (I)," The Journal of the Indian Academy of Mathematics, vol. 11, pp. 121-133, 1989.

[9] M. G. Bin-Saad and J. A. Younis, "On connections between certain class of new quadruple and known triple hypergeomtric series," Tamap Journal of Mathematics and Statistics, vol. 2019, 2019.

[10] J. A. Younis and M. G. Bin-Saad, "Integral representations involving new hypergeometric functions of four variables," Journal of Fractional Calculus and Applications, vol. 10, pp. 77-91, 2019.

[11] H. M. Srivastava and P. W. Karlsson, Multiple Gaussian Hypergeometric Series, Ellis Horwood Lt1., Chichester, UK, 1984.

[12] A. Erdélyi, W. Magnus, F. Oberhettinger, and F. G. Tricomi, Higher Transcendental Functions, McGraw-Hill Book Company, New York, Toronto and London, 1953.

[13] P. Appell, "Sur les fonctions hypergéeométriques de deux variables," Journal de Mathématiques Pures et Appliquées, vol. 8, no. 3, pp. 173-216, 1882.

[14] H. Exton, "Hypergeometric functions of three variables," Journal of Indian Academy of Mathematics, vol. 4, pp. 113119, 1982.

[15] G. Lauricella, "Sulle funzioni ipergeometriche a piu variabili," Rendiconti del Circolo Matematico di Palermo, vol. 7, no. S1, pp. 111-158, 1893.
[16] K. S. Miller and B. Ross, An Introduction to Fractional Calculus and Fractional Differential Equations, Wiley, New York, NY, USA, 1993.

[17] E. D. Rainville, Special Functions, The Macmillan Company, New York, NY, USA, 1960.

[18] J. Choi and A. Hasanov, "Applications of the operator $H(\alpha, \beta)$ to the Humbert double hypergeometric functions," Computers \& Mathematics with Applications, vol. 61, no. 3, pp. 663-671, 2011.

[19] O. I. Marichev, Handbook of Integral Transforms of Higher Transcendental Functions: Theory and Algorithmic Tables., Halsted Press (Ellis Horwood Limited, Chichester), Wiley, New York, NY, USA, 1982. 